\title{
Mission-level performance verification approach for the Euclid space mission
}

Roland D. Vavrek, René J. Laureijs, Jose Lorenzo Alvarez, Jérôme Amiaux, Yannick Mellier, et al. 


\title{
Mission-level performance verification approach for the Euclid space mission
}

\author{
Roland D. Vavrek ${ }^{* a}$, René J. Laureijs ${ }^{\mathrm{b}}$, Jose Lorenzo Alvarez ${ }^{\mathrm{b}}$, Jérôme Amiaux ${ }^{\mathrm{c}}$, Yannick Mellier, \\ Ruyman Azzollini ${ }^{\mathrm{k}}$, Guillermo Buenadicha ${ }^{\mathrm{a}}$, Gonzalo Saavedra Criado ${ }^{\mathrm{b}}$, Mark Cropper ${ }^{\mathrm{k}}$, Christophe \\ Dabin $^{\mathrm{p}}$, Anne Ealet ${ }^{\mathrm{i}}$, Bianca Garilli ${ }^{\mathrm{f}}$, Anna Gregorio ${ }^{\mathrm{n}, \mathrm{o}}$, Henk Hoekstra ${ }^{1}$, Knud Jahnke ${ }^{\mathrm{j}}$, Martin \\ Kilbinger $^{\mathrm{c}}$, Tom Kitching ${ }^{\mathrm{k}}$, John Hoar ${ }^{\mathrm{a}}$, Will Percival ${ }^{\mathrm{g}}$, Giuseppe D. Racca ${ }^{\mathrm{b}}$, Jean-Christophe \\ Salvignol $^{\mathrm{b}}$, Marc Sauvage ${ }^{\mathrm{c}}$, Roberto Scaramella ${ }^{\mathrm{m}}$, Luis M. Gaspar Venancio ${ }^{\mathrm{b}}$, Yun Wang ${ }^{\mathrm{h}}$, Andrea \\ Zacchei $^{\mathrm{e}}$ and Stefanie Wachter ${ }^{\mathrm{j}}$
}

${ }^{a}$ European Space Agency / ESAC, Villanueva de la Cañada, E-28692 Madrid, Spain

${ }^{b}$ European Space Agency / ESTEC, Keplerlaan 1, 2201 AZ Noordwijk, The Netherlands

'IRFU, Service d'Astrophysique, CEA Saclay, F-91191 Gif-sur-Yvette Cedex, France

${ }^{\mathrm{d}}$ CNRS-UPMC, Institut d'Astrophysique de Paris, 98B Bd Arago, F-75014 Paris

${ }^{\mathrm{e}}$ INAF-Osservatorio Astronomico di Trieste, via G.B. Tiepolo 11, 34131 Trieste, Italy

${ }^{f}$ INAF-IASF Milano, vis Bassini 15, I-20133 Milano, Italy

${ }^{g}$ Institute of Cosmology and Gravitation, University of Portsmouth, Burnaby Road, Portsmouth PO1 3FX, UK

${ }^{h}$ IPAC, California Institute of Technology, 314-6, 1200 East California Blvd., Pasadena, CA 91125, USA

${ }^{\mathrm{i}} \mathrm{CPPM}$ de Marseille, 163 avenue de Luminy, 13288 Marseille Cedex 9, France

${ }^{j}$ MPIA, Königstuhl 17, D-69117 Heidelberg, Germany

${ }^{\mathrm{k}}$ Mullard Space Science Laboratory, Holmbury St. Mary, Dorking, Surrey, RH5 6NT, UK

${ }^{1}$ Leiden Observatory, Oortgebouw, 2333 CA Leiden, The Netherlands

${ }^{\mathrm{m}}$ INAF-Osservatorio di Roma, via Frascati 33, I-00040 Monteporzio Catone, Italy

${ }^{\mathrm{n} I N A F-I s t i t u t o ~ N a z i o n a l e ~ d i ~ A s t r o f i s i c a, ~ O A T, ~ v i a ~ A . ~ V a l e r i o ~ 2, ~} 34127$ Trieste, Italy

${ }^{\circ}$ University of Trieste, via A. Valerio 2, 34127 Trieste, Italy

${ }^{\mathrm{p}} \mathrm{CNES}, 16$ Avenue Edouard Belin, 31401 Toulouse Cedex, France

\begin{abstract}
ESA's Dark Energy Mission Euclid will map the 3D matter distribution in our Universe using two Dark Energy probes: Weak Lensing (WL) and Galaxy Clustering (GC). The extreme accuracy required for both probes can only be achieved by observing from space in order to limit all observational biases in the measurements of the tracer galaxies. Weak Lensing requires an extremely high precision measurement of galaxy shapes realised with the Visual Imager (VIS) as well as photometric redshift measurements using near-infrared photometry provided by the Near Infrared Spectrometer Photometer (NISP). Galaxy Clustering requires accurate redshifts $(\Delta \mathrm{z} /(\mathrm{z}+1)<0.1 \%)$ of galaxies to be obtained by the NISP Spectrometer.

Performance requirements on spacecraft, telescope assembly, scientific instruments and the ground data-processing have been carefully budgeted to meet the demanding top level science requirements. As part of the mission development, the verification of scientific performances needs mission-level end-to-end analyses in which the Euclid systems are modeled from as-designed to final as-built flight configurations. We present the plan to carry out end-to-end analysis coordinated by the ESA project team with the collaboration of the Euclid Consortium. The plan includes the definition of key performance parameters and their process of verification, the input and output identification and the management of applicable mission configurations in the parameter database.
\end{abstract}

Keywords: Euclid, performance verification, parameter database, Mission Database

* Euclid Deputy Project Scientist, rvavrek@sciops.esa.int, phone +34 918131398, http://sci.esa.int/euclid

Modeling, Systems Engineering, and Project Management for Astronomy VII, edited by George Z. Angeli, Philippe Dierickx, Proc. of SPIE Vol. 9911, 991105

(C) $2016 \mathrm{SPIE} \cdot$ CCC code: $0277-786 \mathrm{X} / 16 / \$ 18 \cdot$ doi: $10.1117 / 12.2233015$ 


\section{INTRODUCTION}

The primary objective of cosmological research in the coming decade is to understand the accelerated expansion of the Universe. For this purpose, the Euclid mission has been selected for implementation in 2012, it is the second mediumclass mission (M2) of the ESA Cosmic Vision 2015 - 2025 program. The mission is designed to make the most accurate measurement of the expansion history and the growth rate of cosmic structures generated by the accelerated expansion of the Universe, allowing us to make decisive statements on the nature of dark energy. Euclid is a space-borne $1.2 \mathrm{~m}$ survey telescope, its science payload is equipped with two imaging and spectroscopic instruments operating in the visible (VIS) and near-infrared wavelength ranges (NISP). This instrumentation is capable to explore how the accelerated expansion manifests by measuring shapes and redshifts of galaxies over a large fraction of the sky. The satellite is scheduled for launch in 2020 by a Soyuz ST-2.1B rocket and transferred to the L2 Sun-Earth Lagrange point for a six-year mission that will cover at least 15000 square degrees of sky. Euclid will measure more than 1.5 billion galaxy shapes through imaging, and more than 25 million galaxy redshifts through slitless spectroscopy.

This paper is organized as follows: this introductory section summarizes the science case and the mission concept, describes the science payload and the sky survey design in brief. Section 2 describes the allocation of key performances at mission level. Section 3 summarizes the mission-level performance verification concept applied to the Mission Preliminary Design Review in 2015 and will be applied for the Science Performance Verification in 2017-2019. Section 4 describes the Euclid Mission Database (MDB), a centralized mission configuration management system. Finally Section 5 summarizes on the ongoing Euclid performance verification activities and outlook activities in the future.

In the current "concordance cosmological model" cosmic structures of Universe grew hierarchically from gravitational instabilities to form galaxies, galaxy clusters and filaments, three quarters of its mass-energy density at our epoch is in the form of Dark Energy (DE), and approximately one fifth of Dark Matter (DM). While the remaining only five percent composes the source of all visible matter, no existing theories can explain the nature of dark energy and dark matter in framework of the standard cosmological model and the standard model of particle physics.

Euclid is designed to carry out a large area optical and near-infrared imaging survey and a massive spectroscopic survey in the wavelength range $1.1-2.0$ micron. The mission is optimised for two cosmological probes: Weak Lensing (WL) and Galaxy Clustering (GC). These probes enables unprecedented statistical precision for Euclid, and provide a crucial cross-check of systematic effects, which become dominant at these levels of precision. Euclid aims at improving the dark energy Figure of Merit (defined by FoM $=1 /\left(\Delta w_{\mathrm{p}} \times \Delta w_{\mathrm{a}}\right)$, where $w_{\mathrm{p}}$ and $w_{\mathrm{a}}$ parametrize the dark energy equation of state). From the weak lensing and galaxy clustering information alone a FoM of $\sim 430$ can be derived, which is a factor $\sim 20-40$ higher than what is presently achievable by merging all known observations. The following key questions can then be addressed by Euclid:

- Nature of Dark Energy: Is dark energy simply a cosmological constant, or is it a field that evolves dynamically with the expansion of the Universe?

- Modified Gravity: Alternatively, is the apparent acceleration instead a manifestation of a breakdown of General Relativity on the largest scales, or a failure of the cosmological assumptions of homogeneity and isotropy?

- Dark Matter: What is dark matter? What is the absolute neutrino mass scale and what is the number of relativistic species in the Universe?

- Initial Conditions: What is the power spectrum of primordial density fluctuations, which seeded large-scale structure, and how deviations to Gaussian probability distribution in Euclid data can unveil inflation?

Euclid's two primary dark energy probes, GC and WL, are complemented by a number of additional cosmological probes which further improve Euclid's constraining power: cross correlation between the cosmic microwave background and the large scale structure; abundance and properties of galaxy clusters and strong lensing and possible luminosity distance through supernovae Ia.

Euclid will be one of the most powerful tools to test these new theories, one in which the systematics will be controlled to an unprecedented accuracy through the combination of technical capability and different cosmological approaches. 
The main task of the VIS instrument is to enable WL estimates, which require an exquisite image quality to perform accurate galaxy shape measurements. The gravitational potential aggregates ordinary and dark matter as the Universe expands. The complex self-similar interwoven structure of overdensities perturb the path of photons traveling through space from distant background galaxies to the observer. These background objects appear distorted - at small spatial scales comparable to the apparent size of a galaxy - the weak distortion accounts for a small but measurable change in their ellipticity. From the statistical averages of at least 30 galaxy shapes per square arcminute a sufficiently dense galaxy shear pattern can be obtained. When combined with the photometric redshift of the same objects the 3dimensional mass distribution, corrected from the contamination by intrinsic galaxy alignment, can be reconstructed. This is how Euclid measures dark matter distribution. From the mass distribution as a function of redshift, the rate at which matter aggregated can be derived. This is directly related to the expansion rate of the Universe and the growth rate of structure in the Universe, which are attributed to dark energy and dark matter, so the characteristics of the dark energy can consequently also be constrained.

The VIS instrument focal plane array (FPA) consists $6 \times 6$ CCDs $(4 \mathrm{k} \times 4 \mathrm{k}$ pixels each) with 0.101 arcsec pixel platescale, giving a field of view $0.55 \mathrm{deg} 2$ with one wide visible band covering the broad the range $\sim 550-900 \mathrm{~nm}$. VIS is equipped with a shutter mechanism to close the optical path for read out and dark calibration, and a calibration unit for multicolour flat-field measurements. The VIS channel measure the shapes of galaxies with about $0.16 \operatorname{arcsec}(\mathrm{FWHM})$ system point- spread function excluding pixelisation. A detailed description of the VIS instrument can be found in Cropper et al [3] (these proceedings).

The clustering properties of galaxies with redshift is a sensitive alternative and complementary probe of the accelerated expansion of the Universe. The NISP instrument is optimised to characterize galaxy clustering by measuring the $\mathrm{H} \alpha$ (rest wavelength $656.3 \mathrm{~nm}$ ) line redshift of more than 25 million emission line galaxies in the range $0.8<z<1.8$. Redshifts are used to reconstruct the three-dimensional distribution of galaxies in the Universe, which can be quantified in terms of its power spectrum. The amplitude, shape and anisotropy of these statistics contain the crucial information on the expansion and structure growth histories of the Universe.

The NISP instrument contains an array of $4 \times 4$ HgCdTe NIR detectors $(2 \mathrm{k} \times 2 \mathrm{k}$ pixels each) with 0.3 arcsec per pixel and $2.3 \mu \mathrm{m}$ cut-off wavelength. NISP can be operated in either photometer mode or slitless spectrometer mode by means of a filter wheel and a separate grism wheel. In the slitless spectrometry mode, the light is dispersed by a grism in the wavelength range 0.92 to $1.85 \mu \mathrm{m}$ with a constant $\Delta \lambda$ at a spectral resolution $\lambda / \Delta \lambda>380$ for an object of $0.5 \operatorname{arcsec}$ diameter. A detailed description of the NISP instrument can be found in Maciaszek et al. [4] (these proceedings).

To achieve the exquisite science objectives, Euclid must carry out a survey of over $15000 \mathrm{deg}^{2}$ of the extragalactic sky. This survey is complemented by at least $40 \mathrm{deg}^{2}$ of deep fields and calibration observations regularly interleaved within the main survey schedule. The nominal field observation sequence allows to reach VIS imaging of galaxy shapes down to $\mathrm{AB}$ magnitude 24.5 (10 $\sigma$, extended source), NISP photometry in the $\mathrm{Y}, \mathrm{J}$ and $\mathrm{H}$ near-infrared bands $(0.92-2.0$ microns) reaching $\mathrm{AB}$ magnitude $24(5 \sigma$, point source) corresponding to a photometric redshift accuracy of $\sigma(\mathrm{z}) /(1+\mathrm{z})<0.05$. Parallel to the VIS exposures, NISP will detect redshifts of at least 1700 galaxies $/ \mathrm{deg}^{2}$ with a completeness higher than $45 \%$ at a detection limit of $2 \times 10^{-16} \mathrm{erg} / \mathrm{s} / \mathrm{cm}^{2}(3.5 \sigma)$ for a typical source of 0.5 arcsec in the wavelength range 1250-1850 $\mathrm{nm}$. The survey coverage avoids high background regions dominated by the diffuse zodiacal light and galactic background. The Reference Survey defines the actual observing baseline, it implements an efficient scheduling scheme fitting within 6 years of operations with minimum amount of scheduling overheads and survey gaps. A detailed description of the Euclid reference survey can be found in Amiaux et al. [9].

Besides studies in cosmology, Euclid will deliver an immense legacy science archive, with optical imaging at 0.2 arcsec spatial resolution to very faint limits (AB magnitude $24.510 \sigma$ ), infrared imaging in three bands to similar limits and only slightly worse spatial resolution, and spectra and redshifts of nearly 100 million sources. A dataset of this size will have huge scientific impact in various fields of astrophysics.

The key features of Euclid are the amount of data that the mission will generate, the heavy processing that is needed to process science products, and the accuracy and quality control which are required at every step in the processing. The Euclid Science Ground Segment (SGS) is designed to process telemetry data, as well as external data from ground based photometry, to obtain the mission data products. The SGS is composed of a number of geographically distributed Science Data Centres (SDCs), in charge of science data processing, production of Level-1 (raw telemetry), Level-2 (calibrated data) and Level-3 (science-ready) science products, simulations, handling and cross-matching of external data from ground-based surveys, and in general all science-driven data processing. The ESA Science Operations Centre 
(SOC) main responsibility is Level-1 production and Quick Look Analysis (QLA). The science data processing can be decomposed in ten Processing Functions (PFs), each of which represents a self-contained processing unit in the pipeline. A given PF is under responsibility of an Organisation Unit (OU) which develops the algorithms in prototype code, performing numerical tests, and comparing the results against specifications. A detailed description science ground segment concept can be found in Pasian et al. [6].

All Euclid data will be publicly released in subsequent data releases after a relatively short proprietary period and will constitute for many years the ultimate survey database for astrophysics.

The overall scientific aims of Euclid have been recently summarized in [5], an overview of the mission design and performances are also available in this proceedings [2] and in the Euclid Red Book [1].

\section{EUCLID MODELING AND KEY PERFORMANCE PARAMETERS}

The Euclid mission top-level science objectives translate into stringent performance requirements allocated at the different elements of the mission architecture. The Mission Requirements Document (MRD) establishes the general set of performance requirements, while the Mission Budget Justification File provides a requirement-by-requirement description of the rationale and a full description of the derivation to lower levels.

The Euclid mission level performance requirements refer to observing capabilities, and can be grouped in the following categories:

- Image quality: requirements on the complete VIS and NISP observing chain Point Spread Function (PSF) characteristics. The precision with which the Euclid visible image sensors can measure the shapes of galaxies is of paramount importance to the success of the mission.

- Sensitivity (radiometric performance): photometric sensitivity achievable for the visible and near infrared channels, and specifies unresolved emission line detection flux limit for the spectrometric channel.

- Spectroscopic performance: slitless spectroscopy performance encompasses purity (proportion of accurate redshift determination) and completeness (ratio of successfully measured targets from the observable galaxy sample).

- Survey performance: survey area covered by the wide and deep surveys, and total time required to complete the coverage.

Each of these elements are explained in detail in the Red Book [1] and collected for Euclid's three instrumental capabilities in the following sub-sections.

The collection and maintenance of the complete set of assumption and justifications for the requirements flow-down is the responsibility of the mission Systems Engineering function. A Model Based System Engineering (MBSE) approach has been implemented to trace requirements of the complex Euclid systems. For each requirement or group of related requirements, snapshots from the Euclid SysML Model are provided to visualize the traceability and dependency, an example is shown on Figure [1]. Further examples and a description of the Euclid MBSE approach is provided in the same proceedings in Alvarez et al. [10]. 


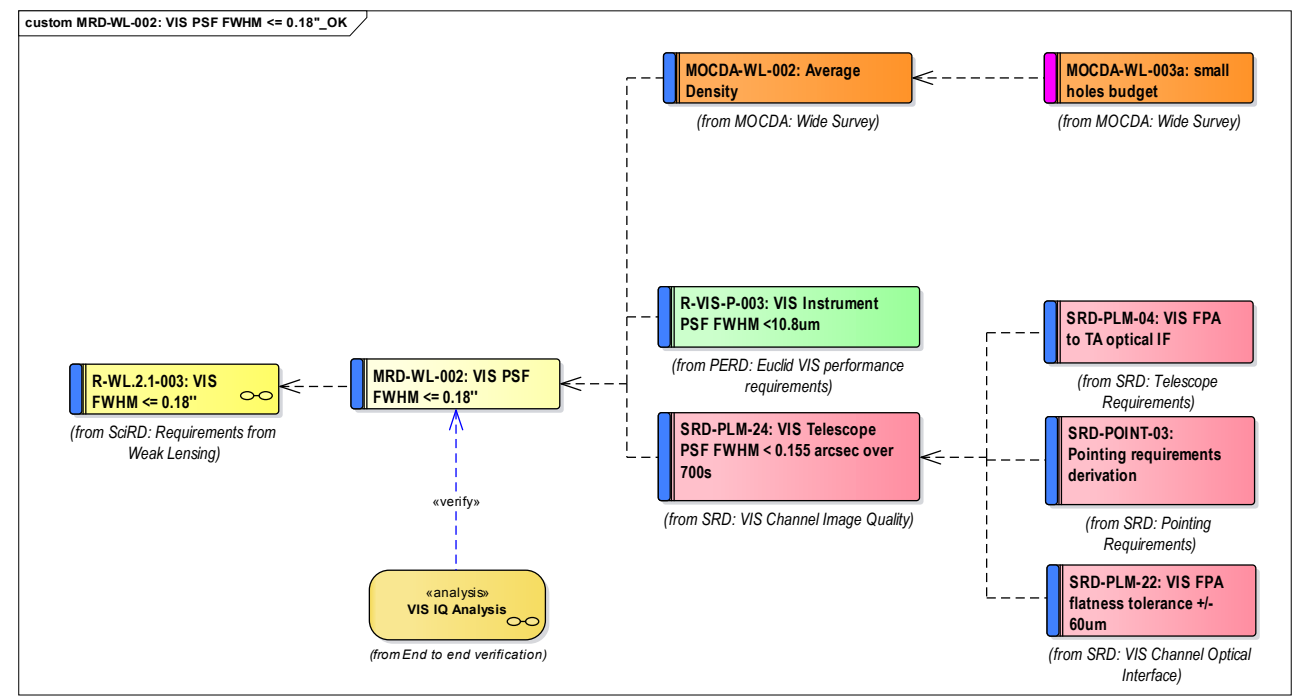

Figure 1 VIS channel PSF FWHM requirements flow-down.

\subsection{Visible imaging}

The main role of the visible imaging is to measure galaxy shapes and hence, the shear induced by weak gravitational lensing as described in the introduction. The observational technique requires a small and stable point spread function, and the uniformity provided by wide field imaging using a stable platform in space. Note that the typical size of a Euclid galaxy is $\sim 0.3$ arcsec. In order to meet the top-level requirements for WL, the visible imaging must:

- Cover a large area of the sky (at least 15000 square degrees) in order to observe a sufficiently large sample of galaxies;

- Have sufficient sensitivity to allow a deep census of galaxies providing a surface density of 30 galaxies per square arcminute;

- Have a PSF which allow determination of shapes down to a fraction of an arcsec. The PSF must be well understood and predictable on any position of the FPA at any time.

The first two are driving the field of view of the detectors and the size of the telescope. The third requirement is the most critical one, and consists of two parts: not only must the Point Spread Function (PSF) be acceptable, but its characteristics must also be known very precisely at any point over the field of view and for any epoch of the observation.

The main requirements on the acceptability of the PSF are on its size expressed in terms of FWHM, its ellipticity ( $\varepsilon$ ), as well as a constraint on the extension of its wings in the secondary lobes $\left(\mathrm{R}^{2}\right)$. Then, the knowledge of this PSF can be expressed in terms of residuals of these quantities (as determined by a model) from their true values. In orbit, the technique adopted by Euclid relies on the availability of a number of relatively bright stars across the focal plane, which allows to calibrate the PSF at these points, and from which a model of the PSF at any position of the focal plane and at the time of the observation can be constrained.

\subsection{Near-infrared imaging}

The NISP photometric measurements completed with ground-based multiband measurements are used to estimate the photometric redshifts of the weak lensing galaxies. Since the main purpose is accurate photometry, the image quality requirements are expressed in terms of radii of encircled energy (EE50, EE80) of $(<0.30,<0.62)$ arcsec, $(<0.30,<0.63)$ 
arcsec and $(<0.33,<0.70)$ arcsec at the centre of the $\mathrm{Y}, \mathrm{J}$ and $\mathrm{H}$ bands respectively. This high image quality is required to meet the sensitivity requirements $\mathrm{AB}$ magnitude 24 ( $5 \sigma$ point source) in all three bands. The $\mathrm{Y}, \mathrm{J}$, and $\mathrm{H}$ band passes are defined for Euclid and instrument and telescope optical design is such that the bands passes are stable and understood at any position of the FPA.

For both visible and infrared imaging, the PSF characteristics of the imaging modes is a convolution the following components: the optical system PSF (generated from the telescope and instrument optical design perturbed according to realistic manufacturing and element misalignment errors), the satellite's pointing jitter, the instrument optics quality, and the detection element response. Extra non-convolutive contribution may also degrade the final PSF.

\subsection{Near-infrared spectroscopy}

The NIR spectroscopic channel is designed to carry out redshift measurements with $\sigma(\mathrm{z}) /(1+\mathrm{z})<0.001$ in several redshift bins up to a redshift of 1.8. As described in the introduction, the redshift measurement relies mainly on the detection of the $\mathrm{H} \alpha$ emission line. For a given $\mathrm{H} \alpha$ line flux, the precision, accuracy and success rate of redshift determination depends on the intrinsic NISP instrumental parameters (PSF size, resolution, and instrumental background) and on the observing strategy adopted to mitigate the specific limitations of the slitless technique, in particular confusion of spectra, out-offield straylight and the Zodiacal light levels. The completeness is defined as the number of galaxies for which a correct redshift is measured at $50 \%$ confidence level, $\left(\mathrm{N}_{\text {correct }}\right)$ divided by total number of galaxies at the flux limit $\left(\mathrm{N}_{\text {meas }}\right)$.

The performance verification has to demonstrate (i) that single redshifts are unbiased and measured with sufficient precision in order to reach the needed flux limit of $2 \times 10^{-16} \mathrm{erg} / \mathrm{s} / \mathrm{cm}^{2}(3.5 \sigma)$ and (ii) that the completeness and purity are sufficient to get galaxy number densities per redshift bins that meet the scientific goal of at least 1700 galaxies/deg ${ }^{2}$. In order to optimize the survey and the unit observing block, it is crucial the relative importance of contributing factors to purity and completeness are well characterized (in particular for signal-to-noise, stellar densities, and number of dispersion orientations parameters).

\subsection{Survey performance}

The survey is intending to repeat a pre-defined sequence of atomic observations over the most suitable extragalactic sky defined by the Wide and Deep survey requirements and associated calibration sequence. The primary requirement for Euclid is the 15000 square degrees area covered by the wide survey. The survey scheduling concept aims at tiling the survey area with the elementary observing pattern in the most efficient way. The total survey time is limited by 6 years.

\section{PERFORMANCE VERIFICATION CONCEPT}

Euclid requires a reliable and executable modelling approach to verify its science return at a few checkpoints where the actual system performance can be synthesized and applied for mission-level predictions.

\subsection{Performance verification cycles}

Euclid mission-level verification activities are distinguished in five components (performances, survey definition, mission profile, mission reliability and interface verification), each component has a specific verification cycle with assigned milestones. Scientific performances are re-evaluated at completion of the End-to-End (E2E) performance analysis cycles, of which four repetitions are foreseen between 2011 and 2020. The first cycle was started after the mission selection in 2012, and the last edition will start after the Mission Critical Design Review (MCDR) in 2018. The planned timeline of Euclid E2E verification cycles is shown on Figure [2]. 


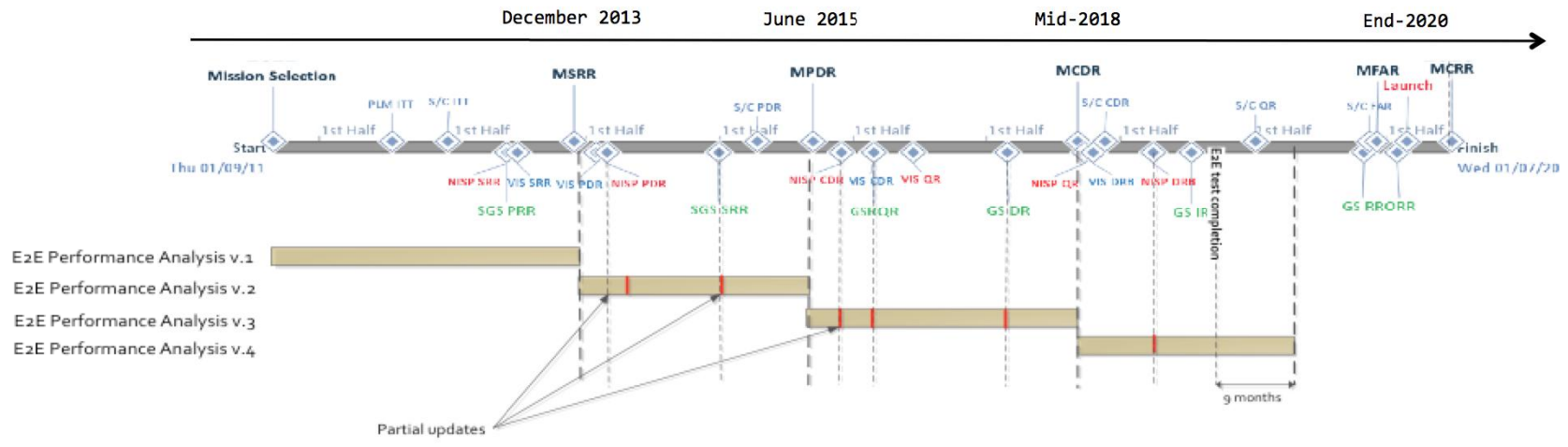

Figure 2 End-to-End performance verification plan

The Euclid mission science objectives translate into stringent performance requirements allocated at the different elements of the mission architecture. The Mission Requirements Document (MRD) establishes the general set of requirements for the Euclid mission. It defines functional and performance requirements to the Euclid system, documents mission constraints and allocates high level interface requirements among the Euclid segments. Key science performance requirements are valid during the Euclid development phase, commissioning and scientific mission operations, and verified through the E2E Performance Verification cycles. The activity tree of E2E performance verification is shown on Figure [3].

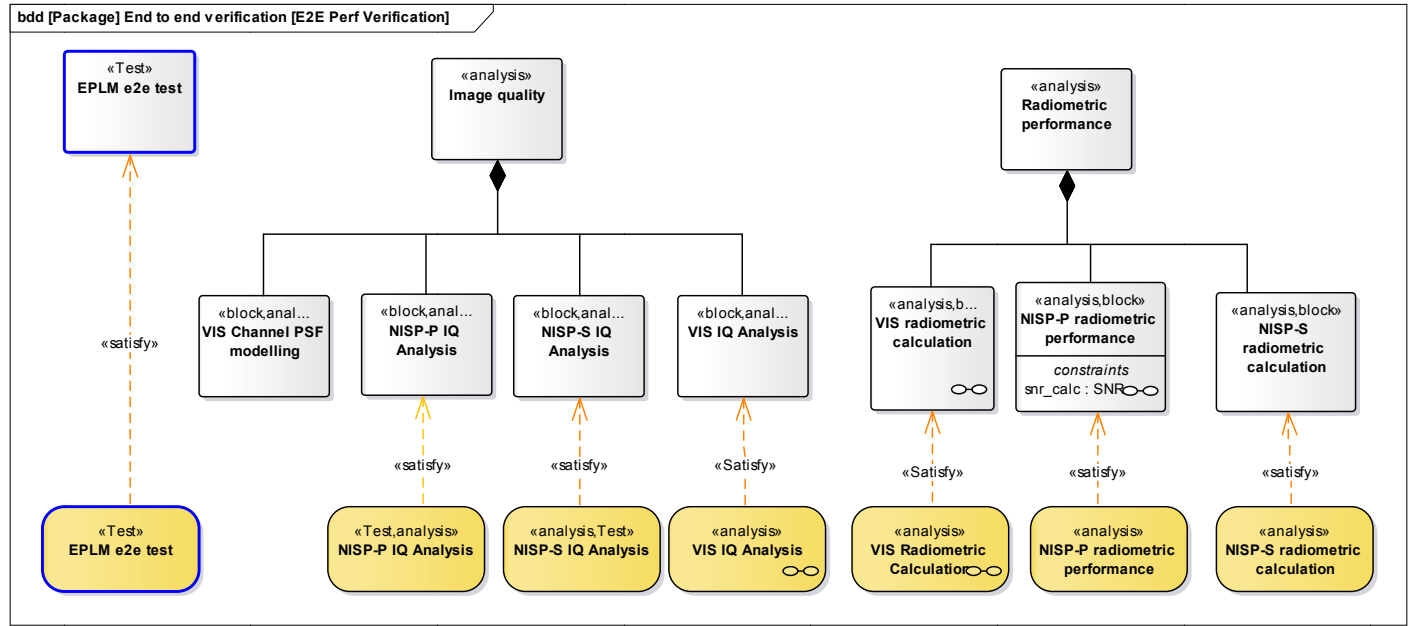

Figure 3 End-to-End performance verification activity tree indicating key performances of observing capabilities

In this section we first summarize mission performances from Mission Preliminary Design Review. The MPDR was concluding the second End-to-End performance verification cycle in 2015, it demonstrates the Level-2 scientific goals can actually be met with current best estimate sub-system performances.

Finally, we outline the validation approach of the Science Performance Validation (SPV) campaigns \#2 and \#3 foreseen in the period of 2017-2019. These two SPVs are considered the most complete science verification efforts for Euclid before launch. The ambitious campaigns aim to verify the Euclid mission is compliant with its top-level cosmology requirements described in the introduction. SPVs are organized and implemented by the Euclid Consortium and supported by ESA, it requires a highly collaborative effort with the involvement of almost all constituents of the Euclid Collaboration. 


\subsection{Performance verification at the Mission PDR}

The performance verifications have been carried out at mission level by combining all individual elements of the mission architecture relevant for the assessment of the exquisite key performances. To evaluate the current performance of the system, analysis performed for all elements where updated information is available from as-designed synthesis. This assessment has been performed in the framework of the Mission PDR and completed in mid-2015. The main science performance verification objectives of the MPDR are: (i) confirm that the mission performance are adequate to fulfill the scientific objectives as specified in the Mission Requirements Document, and (ii) confirm the adequacy of the reference survey definition and its compatibility with the spacecraft design. A detailed description of MPDR results are given in this proceedings in the mission status overview paper by Racca et al. [2].

A central element supporting performance verification activities is the parameter repository. It is imperative that all studies and software development apply consistent assumptions for all elements of the mission. The configuration management of parameters in Euclid Mission Database (MDB) is described in detail in Section 4.

Requirements depending solely on the data processing pipeline performance (for instance non-convolutive bias, PSF modeling residual error), were not analyzed in this cycle because the development of data processing functions is not synchronized to performance verification cycles. These elements will be analysed after the SGS DR, as well as in the EC-led SPV campaigns.

\section{End-to-end performance verification of observing capabilities:}

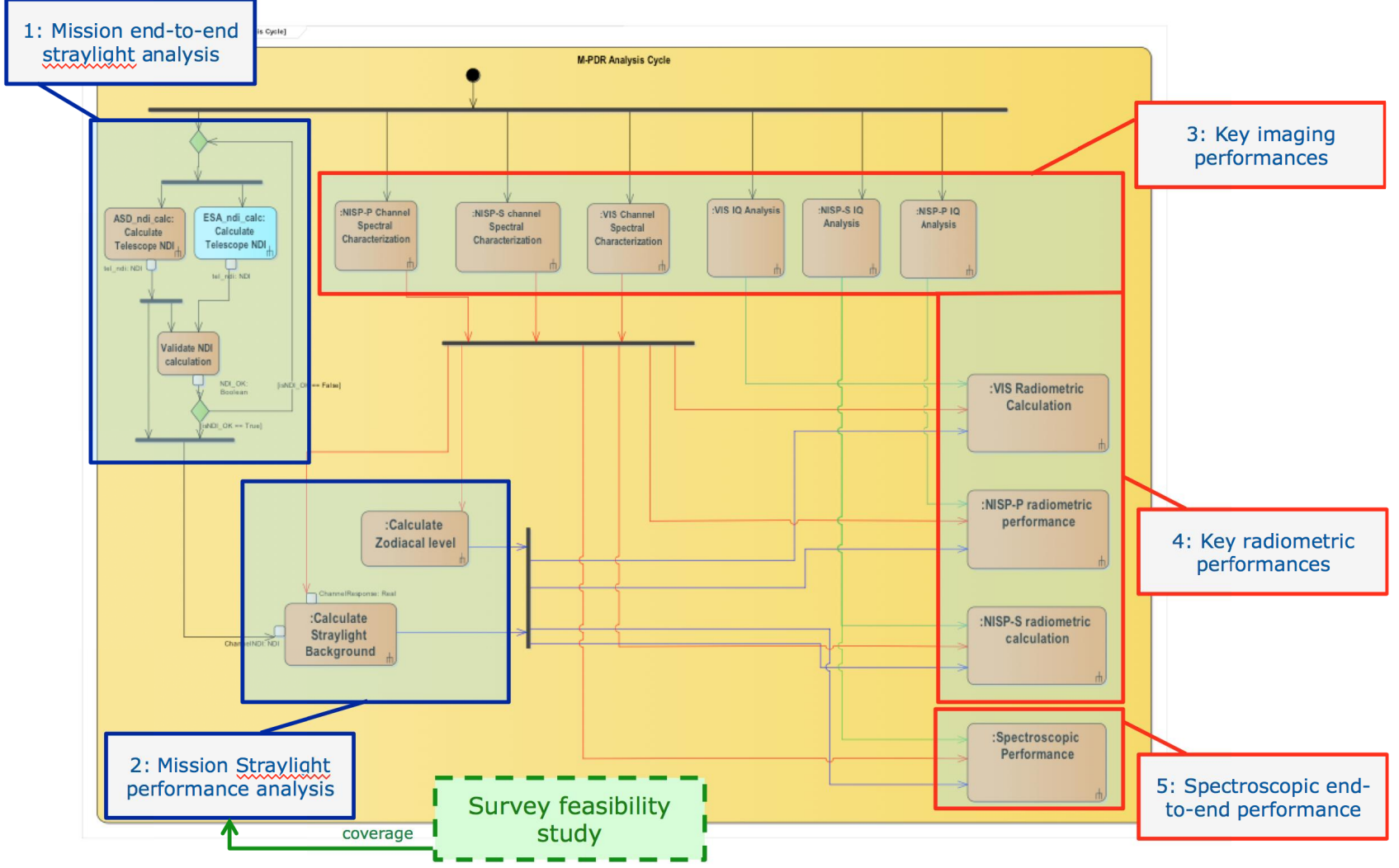

Figure 4 MPDR performance analysis flow diagram 
As shown on the MPDR performance analysis flow diagram (Figure [4]) studies are grouped in five threads:

1. The mission straylight end-to-end analysis provides NDI (Normalised Detection Irradiance) models from an assessment of the straylight performance for both instruments due only to particulate contamination including the telescope plus SVM and instruments VIS and NISP-P optical elements. This assessment compiles the results of simulations performed at ESA and the results presented by ADS and NISP teams at their respective PDRs.

2. Significant attention and effort at system level has been dedicated to the mission straylight performance analysis which provides the estimated total background from straylight and zodiacal light. It provides the reference evaluations of straylight levels covering the in-field and out-field contributions based on the NDI straylight modelling for the spacecraft (telescope plus SVM) and the instruments. Calculations apply stellar surface densities and magnitude distributions combined from 2MASS observations [8] and from the Besancon model of the Milky Way [7] at the faint end required for Euclid sensitivities. Stellar fluxes are integrated at 1 square degree resolution over the reference survey area providing a model of the external illumination of the telescope.

3. Imaging performance analysis calculation is based on a Monte Carlo analysis combining realizations of the optical system at several points in the Field of View (FoV), 100 realizations of pointing jitter series over a nominal exposure, and the best fit CCD PSF determined by Bayesian analysis.

4. Radiometric analysis takes the input from the background radiation determination process of point [2.] above. Illumination is modeled by the input radiance of a reference object combined with background levels. Signal at the detector level is determined by the systems transmission and detection elements quantum efficiency, as well as by the selected radiometric aperture for signal recovery. Analytic calculations combine the derived signal level with detection chain noise contribution and provide SNR maps over the whole reference survey sky coverage.

5. Specific simulations have been carried out for spectroscopy, because of the confusion due to overlapping spectra and the background produced by unresolved faint sources, slitless spectroscopy carries an intrinsic risk of failing to measure galaxy redshifts. The purity and completeness of the baseline redshift recovery method can only be tested through extensive simulations, which have to be as realistic and representative as possible, but also has to be feasible by restricting the sky coverage of computationally very demanding pixel simulations. For the MPDR, 9 pointings on the sky with varying levels of background were selected for performance evaluation representing scenarios of extreme, mean or more frequent skies NISP will observe. The analysis was performed incrementally by including first the effects of straylight, then persistence and finally cosmic rays. The simulation pipeline applied a realistic input catalogue of sources with spectro-photometric information, it created 2D dispersed images, and finally applied spectral extraction and analysis algorithms which produced redshifts. Results of the Mission PDR analysis show that the purity with the current spectra recovery algorithms is limited strongly by the background, in particular by the out-of-field straylight and the zodiacal light levels. It is expected that improvements on data processing, both in terms of background correction and spectral extraction will move performance close to compliance. The next iteration in the Euclid Wide Survey that jointly optimizes the performance of WL and GC should bring the performance to compliance. This analysis highlights the importance on the straylight control both in the design and in the cleanliness and contamination.

Required and estimated Euclid key performances at this analysis cycle are summarized in Table [1].

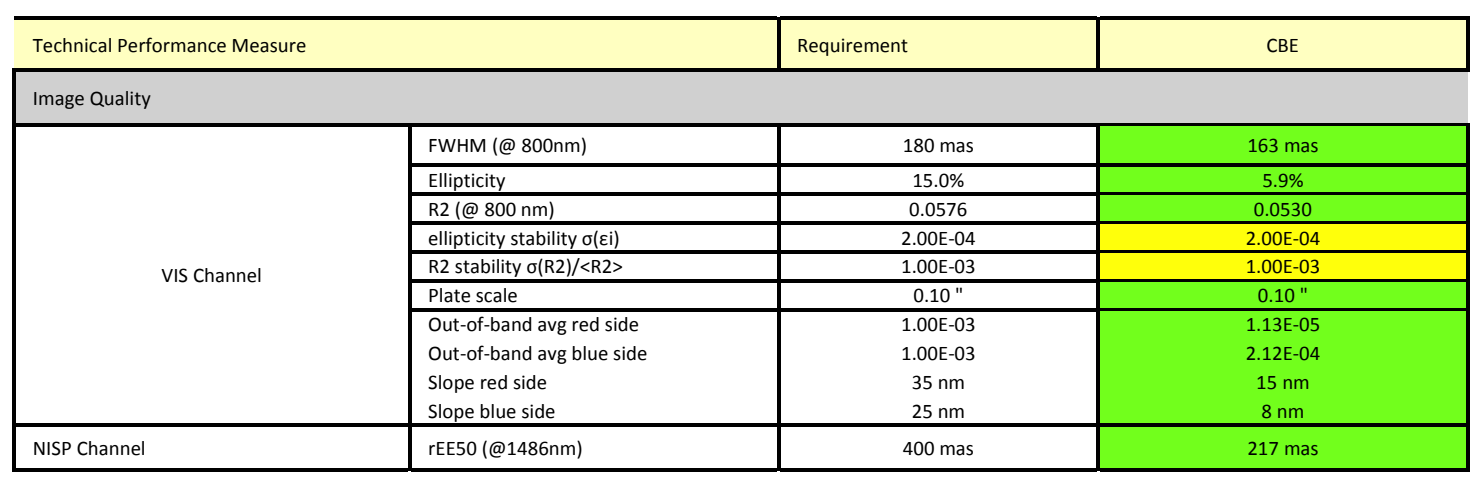




\begin{tabular}{|c|c|c|c|}
\hline & Plate scale & $0.30 "$ & $0.30^{\prime \prime}$ \\
\hline \multicolumn{2}{|l|}{ VIS SNR (for $\mathrm{mAB}=24.5$ sources) } & 10 & 17.1 \\
\hline \multirow[t]{2}{*}{ NISP- P SNR (for mAB = 24 sources) } & J-band & 5 & 6.69 \\
\hline & $\mathrm{H}$-band & 5 & 5.35 \\
\hline \multicolumn{4}{|l|}{ NISP-S Performance } \\
\hline \multicolumn{2}{|l|}{ Purity } & $80 \%$ & $72 \%$ \\
\hline \multicolumn{2}{|l|}{ Wide Survey Coverage } & 15,000 deg 2 & 15,000 \\
\hline \multicolumn{2}{|l|}{ Survey length [years] } & 5.5 & 5.4 \\
\hline
\end{tabular}

Table 1 Euclid system performance summary at Mission PDR

\section{Survey performance verification:}

The Reference Survey (RS) for MPDR demonstrates that the space segment meets the primary science goal of the Euclid mission. The $15000 \mathrm{deg}^{2}$ sky coverage including the deep field and calibration observations can be obtained in less than 6 years of operations $(<5.5$ years for nominal 4400 seconds common block duration). The survey schedule implementation (see Figure [5]) respects all boundary conditions of the system (most important pointing, orbital, telemetry, lifetime, and instrument constraints).

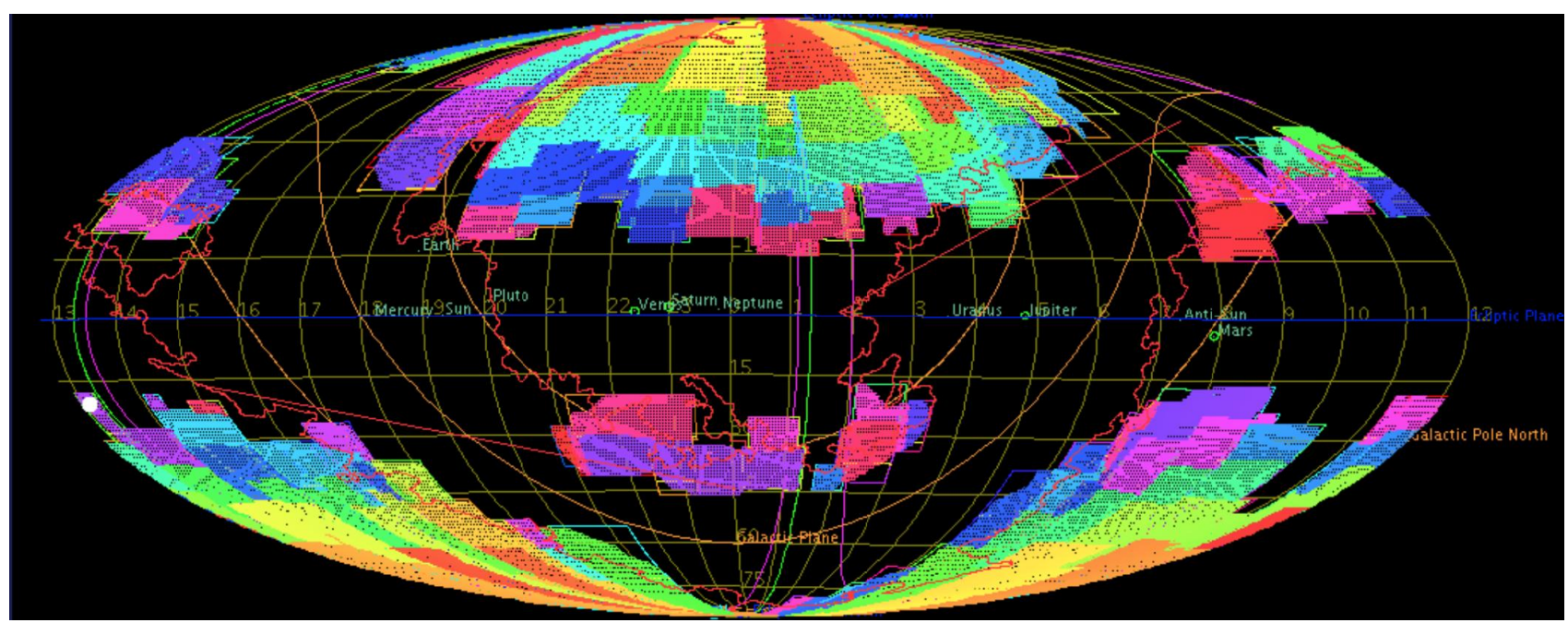

Figure 5 Reference survey coverage in Mollweide projection. Scheduling units (patches) are indicated with colours, where colour coding refers to scheduling order.

In summary, the assessment of mission performance based on the as-designed system configuration shows robust margins on key performances of image quality and radiometric levels. The marginal spectroscopic non-compliance with the purity requirement is expected to be recovered by advancements in capabilities of data processing algorithms, however it reinforces the focus on straylight (and cleanliness) control already in place for the mission. A cartoon of the MPDR performance results are shown in Figure [6]. Difficulty on the y-axis represents a relative number proportional to the difficulty or complexity of the requirement compliance verification process, while the y-axis relative number refers to the maturity of the MPDR performance estimate. The radius of the circles is a third parameter, it is related to the margin at MPDR for the particular parameter. As such, the red circle represents a 'negative radius' (negative margin) in 
case of the spectral purity requirement. The upper-left corner of high difficulty and low maturity indicate requirements which require the most attention in upcoming science verification cycles. These requirements (purity, completeness and spectroscopy SNR) are related to the galaxy clustering probe. With subsequent iteration of E2E performance verification cycles the maturity of estimate will increase for all key performances.

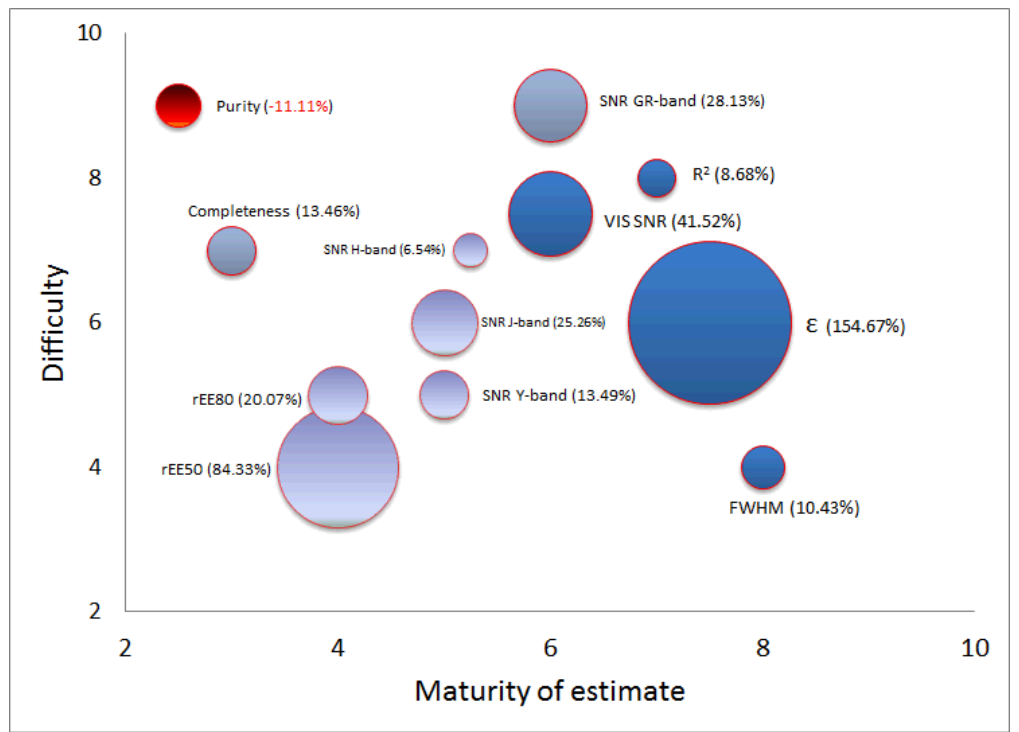

Figure 6 Summary of MPDR key performances on difficulty vs. maturity of estimate diagram

\subsection{Science Performance Verification campaigns}

The successful MPDR is a major achievement for Euclid, however, a need for extension of its objectives and of its methodology has been proposed by the Consortium. The concept of the EC-led Science Performance Verification (SPV) campaigns \#2 and \#3 scheduled in the period of 2017-2019 is outlined in this section. The validation approach of the SPVs requires a very ambitious collaborative effort between the SGS, the Instrument Development Teams, ESA, Industry and the Science Working groups, the outcome is certainly the most reassuring verification that the Euclid mission will meet of its goals originally set up by the scientific community.

The End-to-End performance verification concluded by the MPDR was an ESA Project milestone to evaluate the current performance of the mission. Now that design parameters becomes available and some of the components are being built, a revised and more complete snapshot of mission performances are desirable to obtain. The EC interest is also focused on the verification of top-level science requirements which requires a complete different methodology of simulations and modeling. The two main objectives of the SPV are set up:

1. Verify mission-level performance requirements including mission elements of: joint hardware (instrument and satellite), operations (survey and calibrations) and data processing (SGS pipeline prototype).

2. Verify end-to-end science performance based on the current reference space segment, ground segment and ground data. Verify compliance with top-level (Level-0 and Level-1) science requirements, estimate the Weak Lensing and Galaxy Clustering joint constraining power assuming a reference cosmology.

These two approaches can lead to parallel implementations with different levels of integration in the Euclid infrastructure. It is, however, foreseen the two activities are highly inter-related as described below in a possible scenario currently being adopted.

The first objective is within the ESA verification scope, and to a large extent is in overlap with the objectives of the ESA-led End-to-End science performance verification cycles described in Section 3.1. Although, these are programmatically independent activities, the two campaigns also do overlap in time. One shall consider these are complementary efforts aiming to verify mission-level key performances with different toolsets. Identical boundary 
conditions and parameters will be applied from the same baseline Mission Configuration, therefore the starting point of verifications is considered identical.

The first objective can be achieved by pixel simulations over a large ( $\sim 300$ square degrees) area on the sky. This simulation effort can be implemented through the following procedure:

- Capture the Euclid baseline Mission Configuration in the Mission Database (See Section 4) including current best estimate sub-system performances obtained at CDR (Critical Design Review) level.

- Execute reference cosmology simulation that will be used for both GC and WL probes, create a joint GC and WL all-sky input catalogue (galaxy mockup) with a realistic Universe.

- Use the OU-SIM Processing Function (simulator PF) to generate simulated survey Level 0 data (raw pixel data) for 300 square degrees area on the sky (including VIS, NISP, calibration data plus ground data). These simulations shall be done for a range of scenarios which are defined by a number of simulation configuration parameters:

Cover simulation parameter space what effect radiometric and spectroscopy performance (zodiacal light levels, straylight, extinction, ghosts, PSF and detector effects). These simulated images feed the SGS pipeline in order to estimate the performance of the data processing for variable conditions of background, extinction and star densities. The results of this exercise are directly applicable to mission level key performance verification.

- Use as much of the ground segment pipeline codes to process data and generate data products on which science analysis can be performed. In case a task in a PF does reach the necessary maturity, or the processing can be simplified by simulating residual effects then apply a data processing shortcut. Again, on the processing side a choice of parameters can be set:

Cover data processing parameter space (background subtraction, detector effect correction etc.) and evaluate SNR for point source and galaxies, evaluate spectroscopy processing chain impact on completeness and purity. are applicable to mission level key performance verification.

- Feed the Science Working Group with the measured catalogue and with Level-3 data.

- Establish the catalogue-to-catalogue Euclid transfer function representative of the current estimated Euclid performance and applies to the parameter space covered by the choice of scenarios with all bypasses clearly defined.

The second objective can be achieved if circumventing the computationally very intensive pixel simulations:

- Go from catalogue to catalogue by by-passing applying the Euclid transfer function applicable to the covered parameter space and baseline Mission Configuration

- Feed the science Working Group with the resulting joint catalogue for WL and GC for a reference cosmological model with correct clustering, magnitude, shape parameter, size, redshift, spectrum; all affected by representative post-calibration residuals.

- The SWG establish science performance of Euclid in the current baseline Mission Configuration.

This process is outlined in the chart shown in Figure [7]. The concept can be continuously verified by comparing the output catalogue of pixel simulations with the output catalogue obtained from the bypass for the same set of galaxies.

There are a number of open issues currently being investigated, like what is the most suitable way to calibrate analytical by-pass for shape measurements, photometric or spectroscopic redshifts from first evaluations of the respective Processing Functions. Also need to define clearly the content of pixel simulations in terms of the choice of sky coverage and the precise choice of parameters applied for the scenarios. 


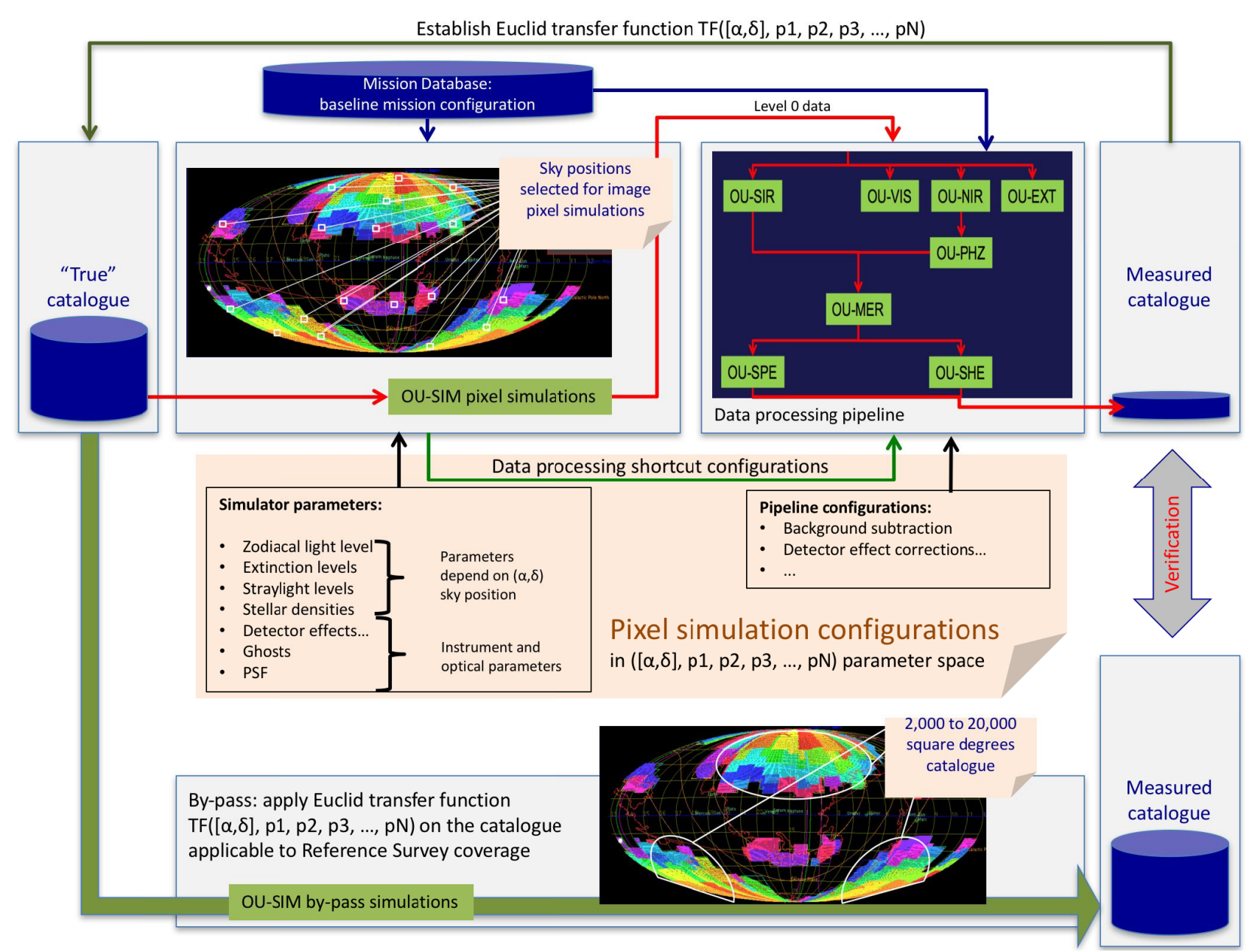

Figure 7 Science Performance Verification model

The SPV cycle \#2 (June 2017) baseline relies on the sub-system CDR information, while for cycle \#3 (April 2019) subsystem flight model (FM) information will be available followed by PLM End-to-End test results and AOCS flight hardware data. Processing Functions apply evolving versions of prototype code, for cycle \#3 a selection of best performing candidate algorithms shall be available.

Besides the parameter baseline and data processing algorithm prototype availability, a necessary precursor of SPV activities is the release of optimized Reference Survey (RS). The optimization considers that the WL and GC probes share the same wide survey area, therefore an optimized coverage strategy has to satisfy both probes with an applicable weighting scheme. The resulting survey area and coverage map has to be created, which is not a trivial task due to the very complex spherical geometry of the survey perimeter. Ultimately, the SPV \#2 analysis shall confirm optimal survey area, and if necessary, the RS will be re-optimised in terms of coverage for SPV \#3.

\section{SYSTEM CONFIGURATION MANAGEMENT}

Numerous teams and hundreds of individuals from industrial contractors, from the Euclid Consortium and from ESA contribute in significant ways to the definition and development of the Euclid systems. It is of critical importance that all teams designing hardware and software sub-systems make identical assumptions on satellite, instrument, mission and natural parameters and apply the most up to date version of them from a central resource. 
In the context of Science Performance Verification, the integration of a unique and controlled snapshot of the mission configuration in both the simulation and data processing software significantly minimize the risk that parameters from a reference configuration may be inadvertently altered. A central repository can guarantee adherence to a reference mission configuration. A large part of the system model applicable to end-to-end pixel simulations will be self-contained within a parameter database, therefore the database content management plays an active role in the science verification process by providing key elements for the simulation and data processing pipeline.

The Euclid Consortium and ESA adopted the highly successful Gaia Parameter Database (GPDB) concept [11] and set out an approach to evolve its design supporting the Euclid mission architecture and the massive data exchange foreseen in the future between the Euclid Mission Database (MDB) and the Euclid Science Ground Segment.

\subsection{System parameters configuration control and distribution system: the Euclid Mission Database (MDB)}

If database client software and users can share the same set of parameters then hard-coding of constants, tables, etc. or inventing numbers from the widespread Euclid documentation is error prone and avoidable. The Euclid Mission Database (MDB) is a response to this problem, the MDB provides a single reference project-wide repository for the Euclid Mission System, it disseminates at any time the official representation of the system consistent with the reference models of each sub-system (for instance, CCD charge transfer inefficiency (CTI) as required specifications, or later flight CCD CTI model characterized in laboratory tests). The MDB provides a centralized version control and distribution system of parameters required by the SGS software, ESA Project, the Euclid Consortium members and Industry contractors. It contains spacecraft and payload parameters, mathematical, physical and astronomical constants and parameters, as well as derived parameters required for the simulation, data processing and calibration processes of the SGS.

MDB follows a hierarchical system engineering top-down decomposition of the satellite and its payload into logical units such as Space Segment, Ground Segment, Launch Segment, Survey, Environment and External Data as shown in an example in Figure [8]. Each of these mission-level components can be followed by additional sub-levels what capture the parameters on which the system is designed and going to be verified. For instance, the Space Segment is decomposed into Instruments, PLM, and SVM, then Instruments are further divided between VIS and NISP. At the level of NISP and VIS the database follows a flat structure in order to avoid multiplication of common parameters (for instance, the number of pixels along the edge of CCDs is identical to all detectors of VIS). The MDB structure implies that parameters are stored exclusively at the lowest level of the hierarchy allowing an easy mapping and location of parameter groups. This becomes a very useful feature when client software needs to locate a given parameter.

Parameter names are designed such that a database user shall be able to alter between database trunks applicable to different mission configurations in a transparent way, without the need to change the reference to individual parameter names.

A Parameter is an abstraction of a configurable item in the database what consists of meta data and a single dataset what can be a text table, fits file, image, binary file etc. A parameter could be a fundamental data item, and derived data; and both of them can be of scalar type or multi-dimensional (such as filter transmission curves as a function of wavelength, or wavefront error maps in the focal plane). Derived parameters can be created by Transfer Functions (TFs), which allow to process some input MDB parameters by a model and produce the output derived parameter. The resulting derived parameters are mandatory to be used by different SGS processing functions. The use of the transfer function ensures consistency in the interpretation of the MDB parameter sets (for instance, the VIS model of non-linearity consists of four parameters and a complex analytic function to derive the output signal, the transfer function implements the analytic function with the given four parameters of the MDB). Transfer functions are stored under the MDB configuration control and accessible by processing functions. Standards about reference systems and related conventions and notations are being collected in order to ensure that parameters can be unambiguously interpreted in the right units, coordinates, angles etc. Some flexibility at the definition of CBE parameters allows cases in which the disseminated value reflects merely a choice rather than an actual measurement or simulated value. These "dummy parameters" are necessary to provide a complete set of parameters required for the proper execution of SGS processing functions. Dummies never obtain validated status, and as the system evolves they are replaced with CBE or as built values. 


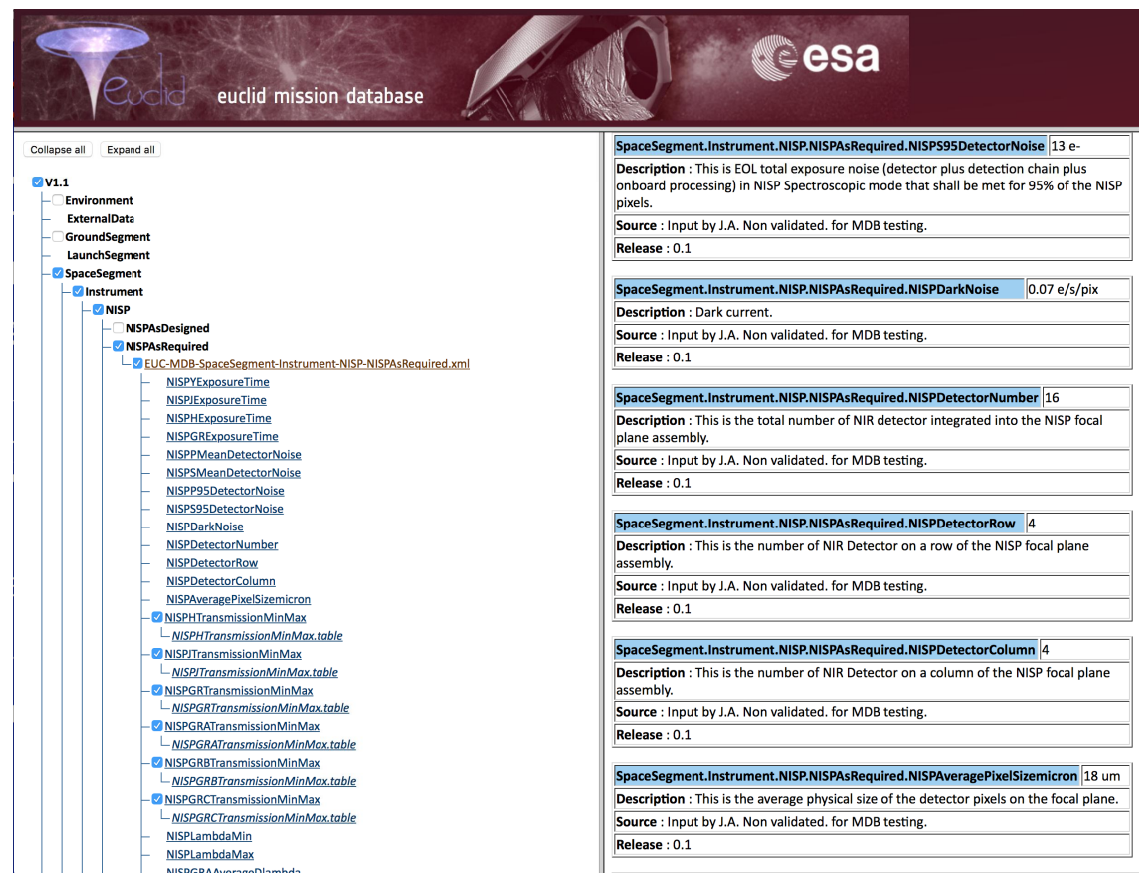

Figure 8 The Euclid MDB online browser interface shows part of the hierarchical decomposition of satellite and payload units (left), and some NISP parameters within the "As Required" mission configuration.

Spacecraft and instrument parameters change with time as design evolves, or as the knowledge of as built system performance advances. The MDB provides parameter branches called Mission Configurations (MCs) what serve to isolate parameter sets. The first mission configuration is "As Required" what is a translation of sub-system requirements into the MDB. It is followed by "As Designed", and "Current Best Estimate" configurations. The final configuration will be "As Built". Each of these database instances adhere to different representation of the system, collected at design milestones, or obtained from unit-tests, sub-system level or ILT or IST test campaigns, or during in-orbit Performance Verification.

Each mission configuration may have a number of database versions, for instance, the current best estimate configuration is continuously updated until parameters applicable to flight-hardware are not available. In practice, parameter values are bound to change in order to facilitate a stable configuration for all applications and activities relying on the MDB in a given development period of the SGS.

MDB is in fact a passive data transfer component serving a number of SGS processing functions, it remains a read-only repository with no automatic data transfer from data processing pipeline and other systems as shown on Figure [9].

The MDB is accessible through two distinct interfaces: (1) a web-based interface accessible from an online browser. Parameter browse capabilities are only foreseen through this web-interface maintained at ESAC; (2) and API interface for offline use, facilitates database access from software clients. Both interfaces provide access to all available fields of a parameter (both meta data and parameter value). The MDB architecture is designed and developed by the Euclid Consortium. 


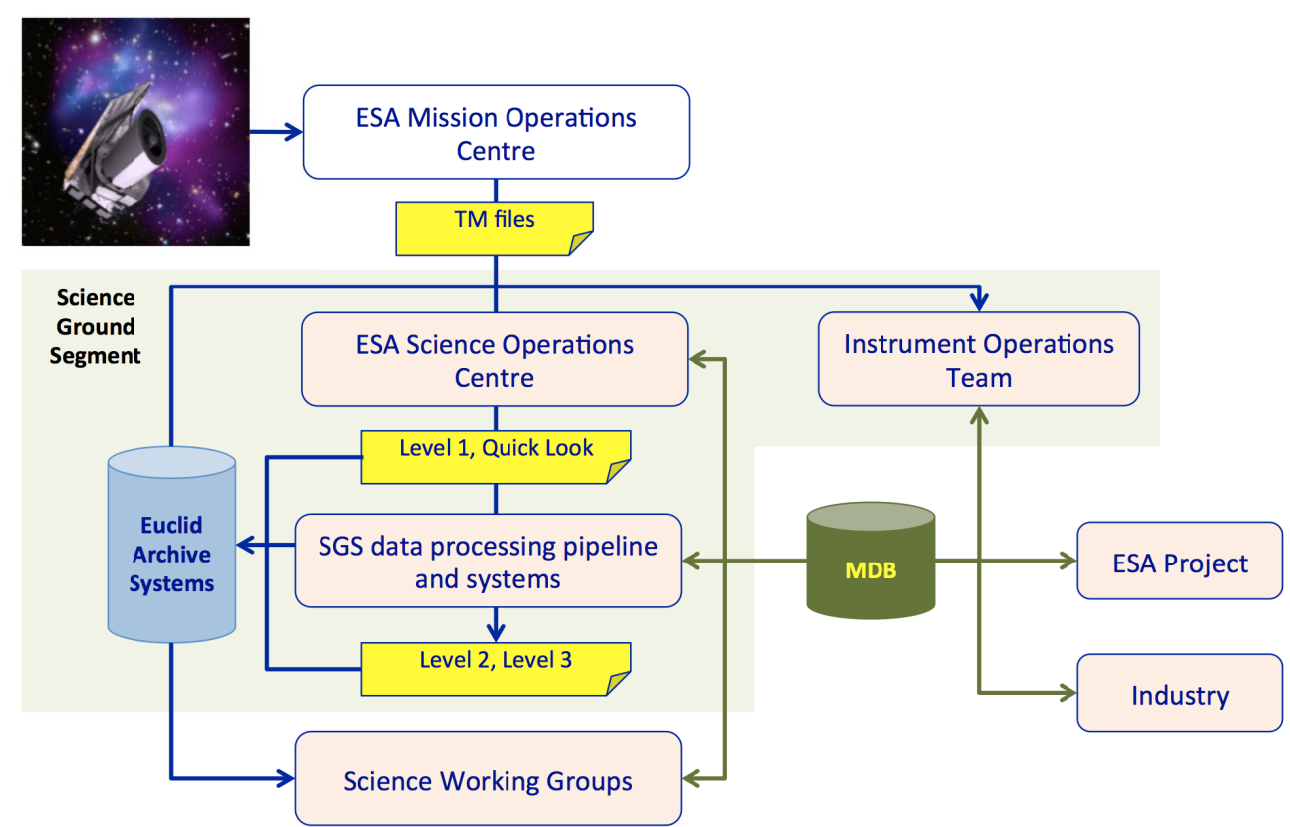

Figure 9 A schematic diagram of the MDB interfaces with the Euclid Science Ground Segment (based on a figure by M. Sauvage presentation "The Euclid Ground Segment”, Lisbon Consortium Meeting 2016)

\subsection{The Mission Database content management process}

A careful control of information flow between parameter providers and parameter users is crucial to ensure that all actors possess a common understanding of parameter values, and parameter required for development are being made available in the MDB on due time. Content management must also ensure the currently best knowledge of the system is properly flown to the MDB.

The MDB management plan promotes a structured sequence of data management activities through a life cycle of data parameters. Activities required to create and change content of the Euclid Mission Database are defined within a configuration control process to ensure the operational database contains only approved and validated items. The risk of distributing unintentionally altered content shall be minimized.

The MDB is managed by a Configuration Control Board (CCB) what applies a continuous supervision over all configurable items of the database.

The MDB CCB is composed of members of the Euclid Mission System Engineering Working Group. Its main duty is the configuration control and validation of parameters deployed in the operational database. The CCB decides when a parameter change request is accepted for implementation in the development database and when a validated operational database version shall be released. System engineers monitor if parameter update is required and provide updated content. The CCB collects parameter justification files (such as test reports which describe the derivation of a given parameter) for traceability of parameter values back to their origin. These sources are referenced in the parameters description or source fields. The CCB assesses and approves delta changes to the database released in the Operational Database, this may happen through a fast track CCB and results a new sub-version. An issue tracking system is used (in Redmine) where MDB users and the CCB can monitor the entire life cycle of database change requests. The system can handle user accounts, issue categories, status and priorities, database version assignments and store parameter justification files. The schematic CCB process is shown on Figure [10]. 


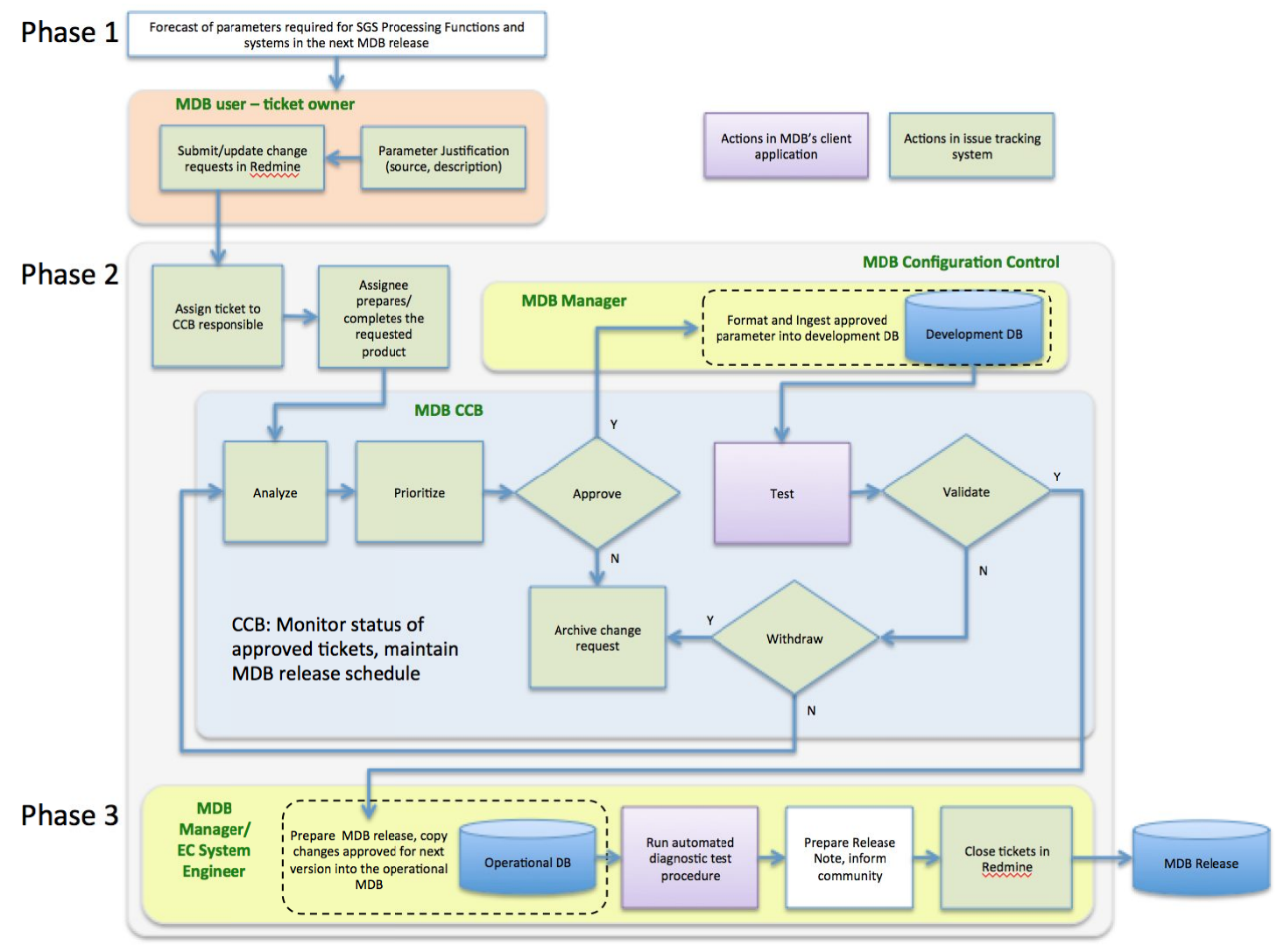

Figure 10 The Euclid MDB configuration control process

The MDB release cycle is synchronized to SGS development cycles, End-to-end simulations campaigns, as well as system design and development milestones. Subsequent MDB releases are foreseen for SGS development milestones called Scientific Challenges (SCs). Scientific Challenges probe the correct functioning of Processing Functions when assembled into a pipeline and integrated in the SGS processing infrastructure. The instrument Critical Design Reviews (CDR) are both expected to be concluded before the end of 2016. The spacecraft CDR is now planned for the first half of 2018. Based on updates from these sub-system level CDRs, the MDB will provide a stable configuration supporting performance evaluation activities for the SPV \#2 as well as activities at mission CDR level, what will be held in mid2018.

\section{CONCLUSIONS}

The Euclid science payload together with the Euclid optical system and survey and calibration strategies will produce high spatial resolution images, photometric measurements and slitless spectra of most of the extragalactic sky, of over 1.5 billion galaxies and a tight control of systematic biases, will provide tight constraints on cosmological models.

The end-to-end science performance verification program is repeated in four cycles, allowing that scientific performances are re-evaluated with the evolving estimate of sub-system performances between 2011 and 2020.

A mission level Preliminary Design Review (MPDR) was successfully held in October 2015. All the mission elements advancement and the mission performance were assessed and considered fulfilling the scientific objectives within the programmatic constraints. 
Following the successful MPDR, the EC-led Science Performance Verification (SPV) plan applicable for the period of 2017-2019 has been set up. The very ambitious SPV aims to verify top-level science performance requirements for the combined WL and GC probes, applying the increasingly mature representation of the mission baseline.

The Euclid Mission Database provides parameter management and distribution system in which the actual system is being modeled and disseminated for the SGS processing functions. This component is essential to synthesize the actual system performances and design parameters for the needs of the ground segment, including science performance evaluations. The hierarchical approach to structure system parameters provide a maintenable, fexible and extensible environment. Mission configurations isolate branches of parameters allowing instrument performances are analysed with as required, as designed, current best estimate or as built parameter sets.

\section{REFERENCES}

[1] Laureijs, R. et al., "Euclid Definition Study report", ESA report ESA/SRE(2011)12, arXiv:1110.3193 (2011)

[2] Racca, G., et al., "The Euclid Mission Design”, Proc. SPIE 9904-19 (2016)

[3] Cropper, M. et al., "VIS: the visible imager for Euclid", Proc. SPIE 9904-20 (2016)

[4] Maciaszek, T., et al., "Euclid Near Infrared Spectro Photometer instrument concept and first test results at the end of phase C", Proc. SPIE 9904-22 (2016)

[5] Amendola, L. et al., "Cosmology and fundamental physics with the Euclid satellite", arXiv:1606.00180 (2016)

[6] Pasian, F., et al., "Science Ground Segment for the ESA Euclid mission", Proc. SPIE 8451 (2012)

[7] A. C. Robin, C. Reylé, S. Derrière and S. Picaud. "A synthetic view on structure and evolution of the Milky Way”, 2003, Astron. Astrophys., 409:523 (2003), erratum: Astron. Astrophys., 416:157 (2004)

[8] Skrutskie M. F. et al., "The Two Micron All Sky Survey (2MASS)", Astrophysical Journal, 131, 1163 (2006)

[9] Amiaux, J.; Scaramella, R.; Mellier et al., "Euclid mission: building of a reference survey", Proc SPIE 8442 (2012)

[10] Alvarez, J. L. et al., "Model-based system engineering approach for the Euclid mission to manage scientific and technical complexity", Proc. SPIE 9911-12 (2016)

[11] Perryman M; de Bruijne, J.; Lammers U. "A parameter database for large scientific projects:application to the Gaia space astrometry mission", Exp. Astron. 22:143-150 (2008) 